\title{
CORRIGENDUM
}

\section{Sleep in dementia and caregiving - assessment and treatment implications: a review}

\author{
D. R. Lee and A. J. Thomas
}

International Psychogeriatrics, FirstView article doi: $10.1017 /$ S1041610210001894

Published online by Cambridge University Press 15 Oct 2010

The authors wish to add the following Acknowledgment:

This work was supported by an award from the U.K. NIHR Biomedical Research Centre for Ageing and Age-related Disease to the Newcastle upon Tyne Hospitals NHS Foundation Trust. 